\title{
Luminescent $\mathrm{PbS}$ and $\mathrm{PbS} / \mathrm{CdS}$ Quantum Dots with Hybrid Coatings as Nanotags for Authentication of Petroleum Products
}

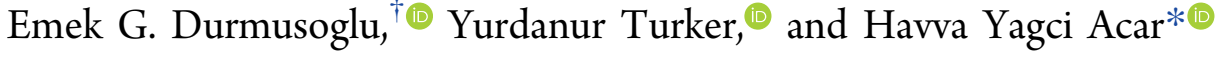 \\ Department of Chemistry, Surface Science and Technology Center (KUYTAM), Koc University, Rumelifeneri Yolu, Istanbul 34450, \\ Turkey
}

Supporting Information

\begin{abstract}
There is an urgent need to tag some valuable liquid products, such as petroleum products, for authentication. However, it is a challenging task because of the strong autofluorescence of liquid petroleum products in the visible range and the chemically active and harsh medium. Therefore, strongly luminescent, near-infrared (NIR) fluorophores with long-term stability are needed. The use of NIR quantum dots (QDs), such as lead chalcogenides, seems to be the best approach; however, most widely used coatings do not provide enough stability, and QDs are quenched in a short time in liquid petroleum products. Here, we report for the first time the synthesis of highly luminescent, ultrasmall, NIR-emitting $\mathrm{PbS}$ and $\mathrm{PbS} / \mathrm{CdS} \mathrm{QDs}$ with a hybrid coating consisting of oleylamine (OLA), 1-dodecanethiol (DT), and poly(methacrylic acid) (PMAA), adopting a simple, greener synthetic method. The photoluminescence (PL) emission wavelengths of these QDs were tuned between 700 and $1100 \mathrm{~nm}$ for detection with low-cost, widely used silicon detectors, which allows easy translation of such QDs as luminescent nanotags to serve as a means for the authentication of goods, such as petroleum. In the nanoparticle design, a thin layer of a CdS shell deposited by a cationexchange process was adopted to enhance the emission intensity and stability of PbS QDs. The influence of postsynthetic ligand exchange of OLA with DT on the stability is also shown. PMAA in the coating provided a significant blue shift in the peak maxima, enhanced the luminescence intensity, and, most importantly, improved the long-term stability of QDs, especially in liquid petroleum products (oil, gasoline, and diesel). Such stability and size tunability was utilized to create binary barcodes. Hence, these QDs are shown as promising luminescent nanotags for liquid petroleum products. The development of such stable QD-based nanotags offers an invaluable use of nanotechnology for optical barcode generation.
\end{abstract}

KEYWORDS: lead sulfide, quantum dot, core-shell, tagging, nanoparticle

\section{INTRODUCTION}

Luminescent quantum dots (QDs) are under heavy investigation for optical tagging as well as for medical optical imaging. $^{1-4}$ Their size-tunable emission wavelength, long photoluminescence (PL) lifetime, broad absorbance, and narrow emission bands make them superior to organic dyes. $^{5-7}$ The latter property of QDs is also extremely valuable when multiple QDs need to be used simultaneously because it allows excitation of different QDs at a single wavelength and the production of an unmixed optical signal. ${ }^{7}$ Such capability is invaluable in optical nanotag generation as well. Optical nanotags may be used in biology and medicine but may even be more practical in tagging valuable goods. Liquid petroleum products such as oil, gasoline, and diesel are such valuable goods. They require a means for authentication and tracking in distribution and in use to ensure originality and to prevent smuggling of these products. ${ }^{8-10}$ A practical optical tag should be added in small amounts, should not hamper the function of the liquid, should be relatively easily detected by an expert, should not be copied easily by nonexperts, and should be stable during the lifetime of the liquid petroleum product. In this case, it may be preferable to have a nanotag that is nonvisible to the naked eye, which favors the use of QDs with near-infrared (NIR) emission. Furthermore, because of strong absorbance in the visible region of the electromagnetic spectrum and autofluorescence of these products up to 700 $\mathrm{nm}$, the use of NIR-emitting QDs would have an additional advantage.

$\mathrm{PbS}$ QDs exhibit strong absorbance in the visible spectrum and emission in the NIR region. ${ }^{11}$ Usually, $\mathrm{PbS} / \mathrm{CdS}$ coreshell structures, wherein the $\mathrm{CdS}$ shell passivates trap states at the nanocrystal surface of the core, are studied for improved emission intensity, stability, and further tuning of the emission wavelength. ${ }^{12-14}$ Thereby, they are popular candidates for photovoltaics, light-emitting diodes, and photodetectors. ${ }^{15-18}$

Received: September 18, 2019

Accepted: November 25, 2019

Published: November 25, 2019 

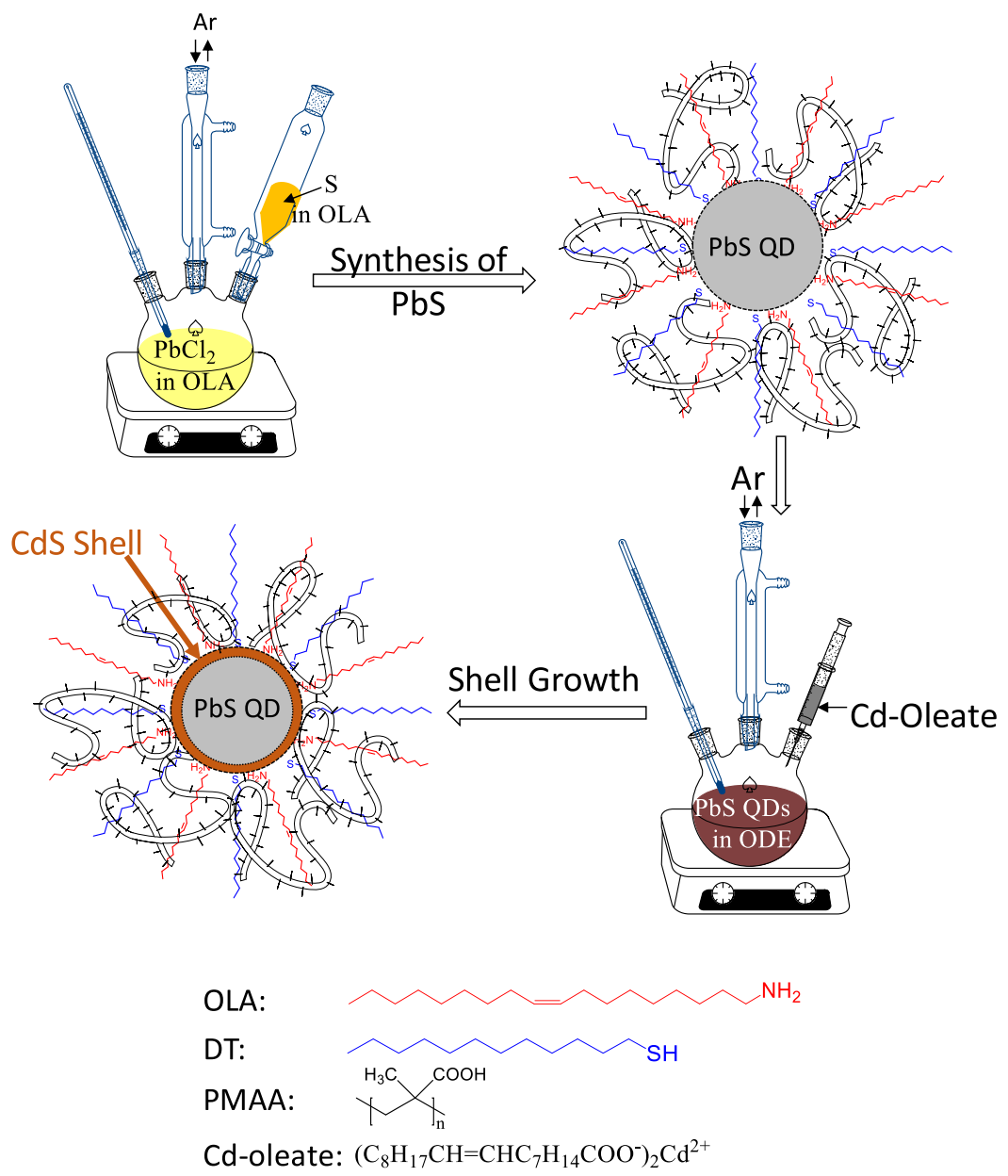

Figure 1. Schematic of the $\mathrm{PbS}$ and $\mathrm{PbS} / \mathrm{CdS}$ QDs synthesis with a PMAA/DT hybrid coating.

In our previous works, a new method for the synthesis of highly luminescent small-sized $\mathrm{PbS}$ and $\mathrm{PbS} / \mathrm{CdS}$ QDs with emission below $1100 \mathrm{~nm}$, which is the detection limit for widely used low-cost silicon detectors, has been reported using nontoxic, nonoxidative, abundant, and low-cost precursors. ${ }^{14,19}$ $\mathrm{PbS}$ QDs were prepared with a mixed oleylamine (OLA) and 1-dodecanethiol (DT) coating, which rendered them more stable in hydrocarbon solvents than the ones reported in the literature; however, they could not resist a slow Ostwald ripening overtime. ${ }^{19} \mathrm{PbS} / \mathrm{CdS}$ core-shell structures produced from these $\mathrm{PbS}$ QDs via room temperature cation exchange improved the emission intensity compared to the core $\mathrm{PbS}$ and provided an opportunity to produce $\mathrm{PbS} / \mathrm{CdS}$ with emission below $800 \mathrm{~nm}{ }^{14}$ These $\mathrm{PbS} / \mathrm{CdS}$ QDs showed improved stability with some red shift in the peak position in chloroform. Yet, they still exhibit a considerable loss of emission intensity over time in petroleum products.

Our group has previously shown that coating QDs with polymers, which have an affinity for the crystal surface and have favorable interaction with the dispersing solvent, does improve the colloidal stability of QDs dramatically. ${ }^{6,20}$ Yet, because of the repeat unit repulsion and thermodynamics of the polymer chain, a dense surface adsorption on the crystal is not possible, and this leaves uncoordinated sites on the crystal surface acting as defects, which causes weak luminescence. ${ }^{20}$ Synergy obtained from the simultaneous use of polymers with small thiolated molecules overcomes this problem. Small molecules diffuse to the crystal surface and passivate the uncoordinated sites. Poly(acrylic acid)/thioglycolic acid (TGA)-coated CdS QDs showed luminescence intensity as strong as that of TGA-coated CdS QDs with excellent colloidal stability over years. ${ }^{20}$

In this work, we aimed to develop QDs with strong emission between 700 and $1100 \mathrm{~nm}$ and with acceptable stability over a month in petroleum products to be used as an optical nanotag for authentication. For this purpose, $\mathrm{PbS}$ and $\mathrm{PbS} / \mathrm{CdS}$ QDs with a hybrid coating composition composed of poly(methacrylic acid) (PMAA) and small molecules, OLA and DT ligands, were prepared. PMAA would provide both carboxylic acid units for adsorption on the nanoparticle surface and solubility in an organic solvent due to the methyl substituent. The influences of the existence of PMAA in the coating and postsynthetic exchange of OLA with DT on the particle properties, as well as reaction variables such as the molecular weight of the polymer, stoichiometry, reaction time, and temperature, were studied. The colloidal and optical stabilities of QDs were monitored in a typical organic solvent (chloroform) and in petroleum products over a period of 1-6 months. Stable particles were used to create binary barcodes as nanotags.

\section{EXPERIMENTAL SECTION}

2.1. Materials. Lead chloride $\left(\mathrm{PbCl}_{2} ; 98 \%\right)$, elemental $\mathrm{S}$ (99.99\%), oleylamine (OLA; technical grade, 70\%), 1-dodecanethiol (DT; 98\%), cadmium oxide (99.5\%), oleic acid (OA; 99\%), 1octadecene (98\%) were purchased from Sigma-Aldrich. Toluene, 
(a)

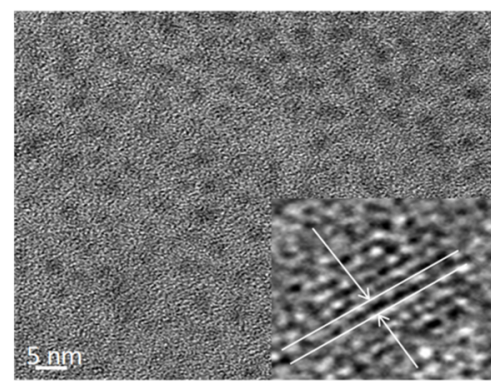

(b)

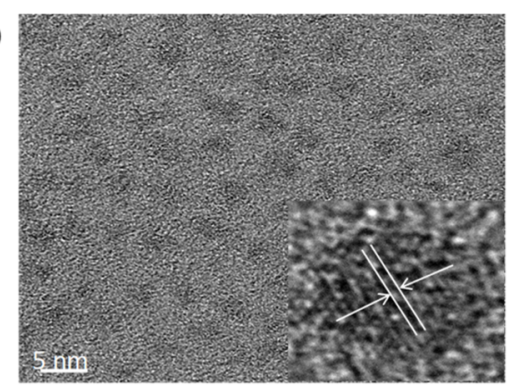

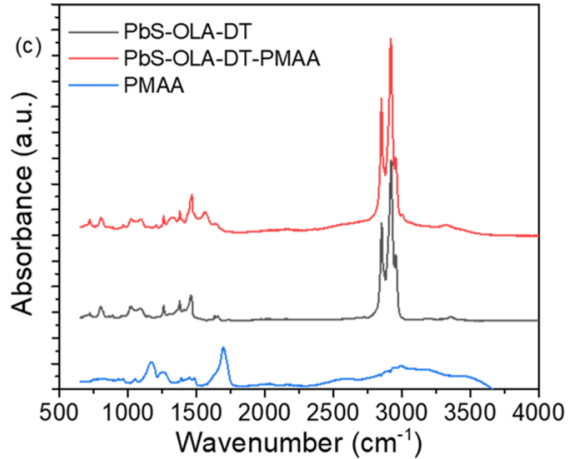
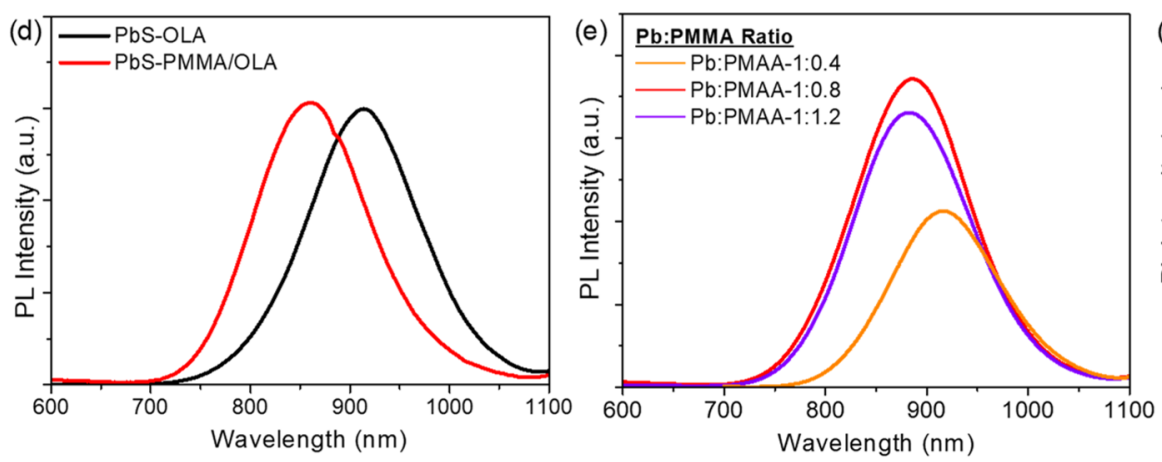

Figure 2. TEM images of (a) PbS and (b) PbS/CdS QDs (insets show the HRTEM images). (c) FTIR spectra of PMAA, PbS-OLA QDs, and PbS-PMAA/OLA. (d) PL spectra of PbS-OLA and PbS-PMAA/OLA. PL spectra of different PbS QDs synthesized at different Pb/PMAA ratios under identical conditions and allowed to grow (e) 1 and (f) $10 \mathrm{~min}$. Pb:S:OLA = 1:0.2:15; reaction temperature $=80{ }^{\circ} \mathrm{C}$; $M_{\mathrm{w}}$ of $\mathrm{PMAA}=131500$ $\mathrm{g} \mathrm{mol}^{-1}$.

chloroform, and ethanol were all "for synthesis" quality and were purchased from Merck. Two types of PMAA were used: (1) $M_{\mathrm{w}}=$ $131500 \mathrm{~g} \mathrm{~mol}^{-1}$, synthesized in our group via free-radical polymerization of methacrylic acid and treated with sodium hydroxide to generate poly(methacrylic acid sodium salt); (2) $M_{\mathrm{w}}=5000 \mathrm{~g} \mathrm{~mol}^{-1}$ was purchased from Sigma-Aldrich.

2.2. Synthesis. Synthesis of Colloidal PbS-PMAA/OLA QDs. The synthesis of OLA/PMAA-coated PbS QDs (PbS-OLA/PMAA) was performed under an argon atmosphere to avoid oxidation. First, the $S$ precursor was prepared by dissolving elemental $S$ (0.008 g, 0.25 mmol) in OLA $(2.5 \mathrm{~mL})$ by sonication under an argon flow for $1 \mathrm{~h}$, followed by vacuum at room temperature for another $1 \mathrm{~h}$. A lead precursor was prepared separately by dissolving $\mathrm{PbCl}_{2}(0.28 \mathrm{~g}, 1$ $\mathrm{mmol}$ ) and the desired amount of PMAA (the amount of PMAA was calculated from the number of repeat units) in OLA $(5 \mathrm{~mL})$ in a three-necked round-bottom flask at $120{ }^{\circ} \mathrm{C}$ under an argon flow, followed by vacuum at this temperature for $1 \mathrm{~h}$. This colorless solution was purged with argon again at $120{ }^{\circ} \mathrm{C}$ for $30 \mathrm{~min}$ and then cooled to the desired particle growth temperature, at which the desired amount of DT (up to $2 \mathrm{~mL}$ in this work) was added. The solution immediately turned clear yellow. This solution was diluted with argon-purged dry toluene $(17.5 \mathrm{~mL})$. Then, a OLA/S solution (2 $\mathrm{mL}, 0.2 \mathrm{mmol}$ of $S$ ) was injected into the $\mathrm{PbCl}_{2} / \mathrm{PMAA} / \mathrm{DT} / \mathrm{OLA}$ solution. After the desired growth time, the reaction was quenched in cold water and cold ethanol was added. The solution was centrifuged at $6000 \mathrm{rpm}$ and precipitated twice, and the QDs were resuspended in chloroform.

Synthesis of Colloidal PbS/CdS-PMAA/OLA QDs. PbS/CdS coreshell QDs were synthesized by the cation-exchange method at the desired temperature (room temperature or $65{ }^{\circ} \mathrm{C}$ in this work) as explained in our previous work (Figure 1). ${ }^{14}$ Precipitated core-shell QDs were annealed in a vacuum oven at $100{ }^{\circ} \mathrm{C}$ in a solid powder form over glass substrates.

DT Ligand Exchange. DT was added to a chloroform suspension of $\mathrm{PbS}$ and $\mathrm{PbS} / \mathrm{CdS}$ QDs and stirred for $15 \mathrm{~min}$ to replace the OLA of the coating at room temperature $[1: 4(\mathrm{v} / \mathrm{v})$ OLA/DT]. The resulting $\mathrm{PbS} / \mathrm{CdS} \mathrm{QDs}(\mathrm{PbS} / \mathrm{CdS}-\mathrm{PMAA} / \mathrm{DT})$ were precipitated in ethanol, removed from the supernatant by centrifugation, and resuspended in chloroform. These QDs were stored in ambient conditions.

Characterization Methods. Absorbance spectra were taken in the range of $400-1200 \mathrm{~nm}$ by a Shimadzu 3600 PC UV-vis-NIR spectrometer. The concentration was calculated from absorbance of the QDs. PL spectra were recorded by a homemade system consisting of a DPSS laser source working at $532 \mathrm{~nm}$, a monochromator $(1 / 8$ Newport Cornerstone 130), and a silicon detector with a 320-1100 $\mathrm{nm}$ working range (Thorlabs PDF10A). A long-pass filter was used that had a transmission of $90 \%$ from 550 to $1100 \mathrm{~nm}$. All recorded data were divided into the responsivity of the detector at each specific wavelength. To eliminate the concentration dependence of PL measurements for an accurate comparison of the PL data of different preparations, the PL data were divided into the absorbance value at the specific excitation wavelength. All provided PL data are absorption-calibrated spectra (normalized to a relative comparison). High-resolution transmission electron microscopy (HRTEM) and scanning transmission electron microscopy (STEM) studies were carried out with a JEOL-ARM 200 cold-field emission gun operating at $200 \mathrm{kV}$. STEM micrographs were taken with a high-angle annular dark-field detector.

\section{RESULTS AND DISCUSSION}

3.1. Development and Characterization of PbS QDs. In the synthesis of $\mathrm{PbS} \mathrm{QDs}$, a greener and low-temperature synthetic method developed previously by our group was adopted. ${ }^{19}$ In this procedure, $\mathrm{PbCl}_{2}$ and elemental $\mathrm{S}$ were used as precursors, a OLA/toluene mixture was used as the solvent, a OLA/DT mixture was used as the capping agent, and particle growth was conducted at low temperatures (below $100{ }^{\circ} \mathrm{C}$ ). Our previous synthetic method was modified with the addition of PMAA to improve the colloidal stability in liquid petroleum products and improve the luminescent properties of $\mathrm{PbS}$ and $\mathrm{PbS} / \mathrm{CdS} \mathrm{QDs}$. In order to obtain small, size-tunable, 

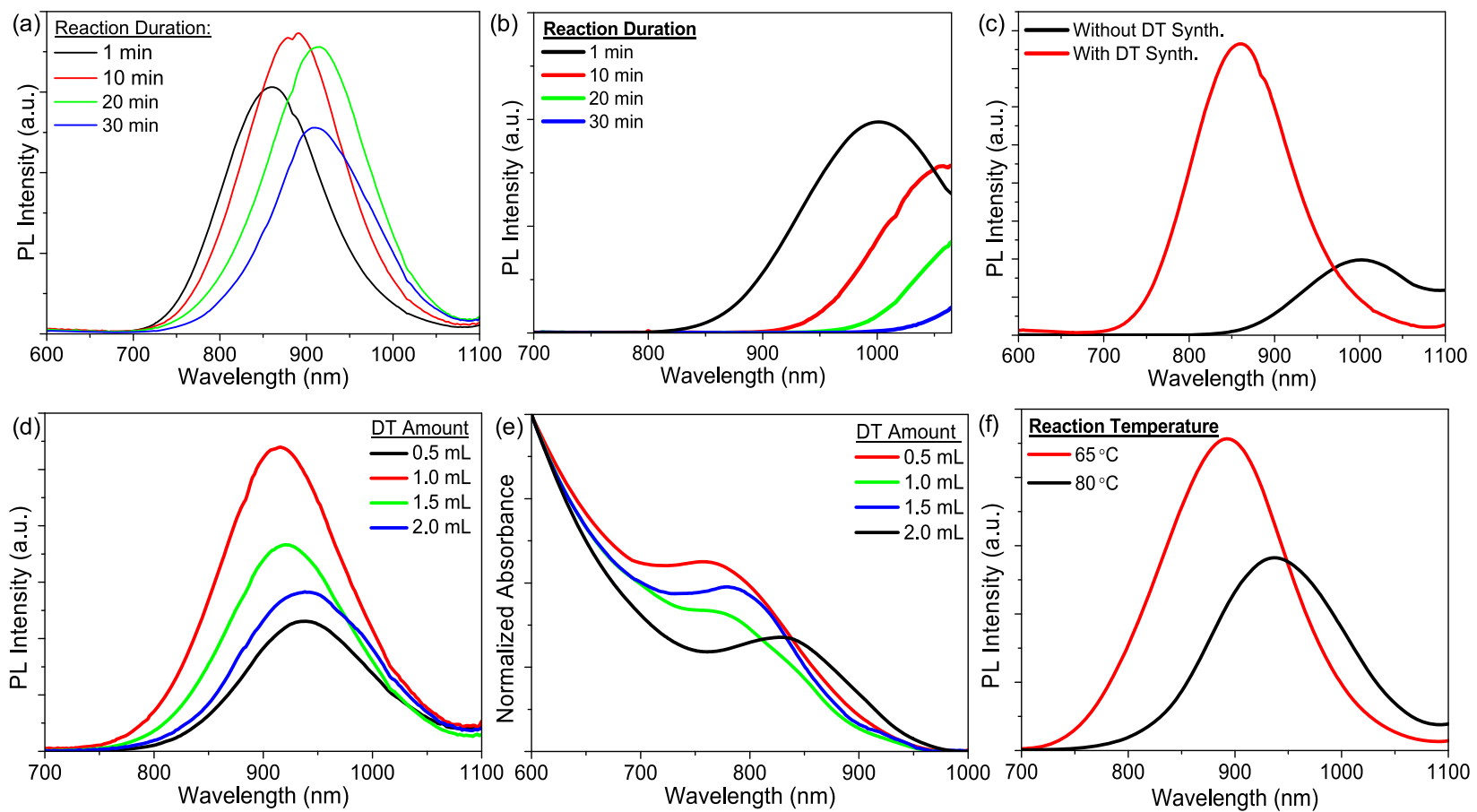

Figure 3. PL spectra of PbS-PMAA QDs produced (a) with $1 \mathrm{~mL}$ of DT and (b) without DT in $30 \mathrm{~min}$ and (c) in $1 \mathrm{~min}$. (d) PL and (e) absorbance spectra of PbS-PMAA prepared with different DT amounts. (f) PL spectra of PbS-PMAA QDs prepared with $0.5 \mathrm{~mL}$ of DT at different reaction temperatures.

monodispersed $\mathrm{PbS}$ QDs, a diffusion-controlled growth technique, which is more suitable for industrial-scale production, was employed. ${ }^{21-23}$ TEM images indicate the formation of monodispersed, quasi-spherical $\mathrm{PbS}$ with ca. $3 \mathrm{~nm}$ diameter (Figures 2a and S1a,b). The smallest particle produced with the original method has the first excitonic peak at $950 \mathrm{~nm}(3.70 \mathrm{~nm}$ particle size $) .^{22}$ HRTEM images in the inset of Figure 2a,b show the crystal lattice fringes of $\mathrm{PbS}$ and $\mathrm{PbS} / \mathrm{CdS}$ QDs with spacings of 0.219 and $0.214 \mathrm{~nm}$, respectively. These values match well with the interplanar distance of the (220) plane of PbS (Figure S1). Furthermore, lattice indices identified from the X-ray diffraction pattern confirm the cubic rock-salt crystal structure of $\mathrm{PbS}$ (Figure S2).

The presence of PMAA in the coating of these PbS QDs was confirmed with Fourier transform infrared (FTIR) analysis (Figure 2c). The peaks at 1552 and $1380 \mathrm{~cm}^{-1}$ belong to $\mathrm{C}=$ $\mathrm{O}$ and $\mathrm{C}-\mathrm{O}$ stretching of the carboxylic acid groups. The strong $\mathrm{C}=\mathrm{O}$ stretching band of the carboxylic acid at 1700 $\mathrm{cm}^{-1}$ was shifted to $1630 \mathrm{~cm}^{-1}$ as a result of bonding with surface $\mathrm{Pb}$ atoms, indicating interaction of the polymer with the crystal surface. ${ }^{24}$

Influence of PMAA on the Particle Properties. For simplicity in naming, QDs with a OLA/DT mixed coating will be called PbS-OLA and QDs with OLA/DT/PMAA will be called PbS-PMAA/OLA (see the Supporting Information for a list of different QDs with various ligand combinations). In order to evaluate the influence of PMAA on the optical properties, $\mathrm{PbS}$ QDs were prepared with and without PMAA under identical conditions $\left(1 \mathrm{~min}\right.$ reaction duration at $80{ }^{\circ} \mathrm{C}$ with a $\mathrm{Pb}: \mathrm{PMAA}$ ratio of 1:0.4). Interestingly, the addition of PMAA did not cause any significant difference in the emission intensity (Figure 2d) but about a $54 \mathrm{~nm}$ blue shift in the emission maxima from 913 to $859 \mathrm{~nm}$. This shift corresponds to roughly a $\sim 0.15 \mathrm{~nm}$ size difference (see the Supporting
Information for the size calculation). PMAA complexes $\mathrm{Pb}^{2+}$ ions in solution, so each polymer chain may be considered as a nanoreactor as well. As the amount of PMAA increases, there will be less $\mathrm{Pb}^{2+}$ ions per polymer chain. If these $\mathrm{PMAA} / \mathrm{Pb}^{2+}$ complexes are considered like nucleation sites, smaller particles will be produced at smaller $\mathrm{Pb}$ :PMAA ratios. In addition, these nanoreactors would have lower mass-transfer rates as well. Parts $e$ and $f$ of Figure 2 show the difference in the luminescence of QDs, produced in 1 and $10 \mathrm{~min}$ reactions, respectively, using different $\mathrm{Pb}: \mathrm{PMAA}$ ratios. Increasing the amount of PMAA in the coating composition at constant lead concentration causes a blue shift in the emission wavelength. Although the difference between Pb:PMAA ratios of 1:0.8 and $1: 1.2$ is not significant ( 858 and $870 \mathrm{~nm}$, respectively) in a 1 min reaction, their PL emission peak positions and intensities are significantly different from those of the Pb:PMAA $=1: 0.4$ ratio $(951 \mathrm{~nm})$. This indicates that emission or size tuning with PMAA amounts beyond a Pb:PMAA ratio of $1: 08$ is not possible. On the other hand, the emission intensity of $\mathrm{PbS}$ PMAA increased roughly 3-fold and 4-fold as the Pb:PMAA ratio was reduced to $1: 0.8$ and $1: 1.2$, respectively, in $1 \mathrm{~min}$ reactions. The PL emission intensity of QDs grown for $10 \mathrm{~min}$ shows the same trend with less difference. This indicates that there is an optimum $\mathrm{Pb}: \mathrm{PMAA}$ ratio that provides the best possible surface passivation and size control. Higher amounts of carboxylic acid units along with smaller crystal size may bring about better surface passivation.

The time-dependent emission profiles of $\mathrm{PbS} \mathrm{QDs}$ are shown in Figure S3a,b. The particle growth mechanism has been explained with Ostwald ripening, wherein smaller particles dissolve and join to bigger particles as the reaction continues to reduce their surface energy. ${ }^{11,22,25,26}$ However, we have observed a blue shift in the emission peak of $\mathrm{PbS}$ prepared at $\mathrm{Pb}: \mathrm{PMAA}$ ratios of 1:0.4 with increasing intensity throughout the $30 \mathrm{~min}$ growth period. This may be explained 

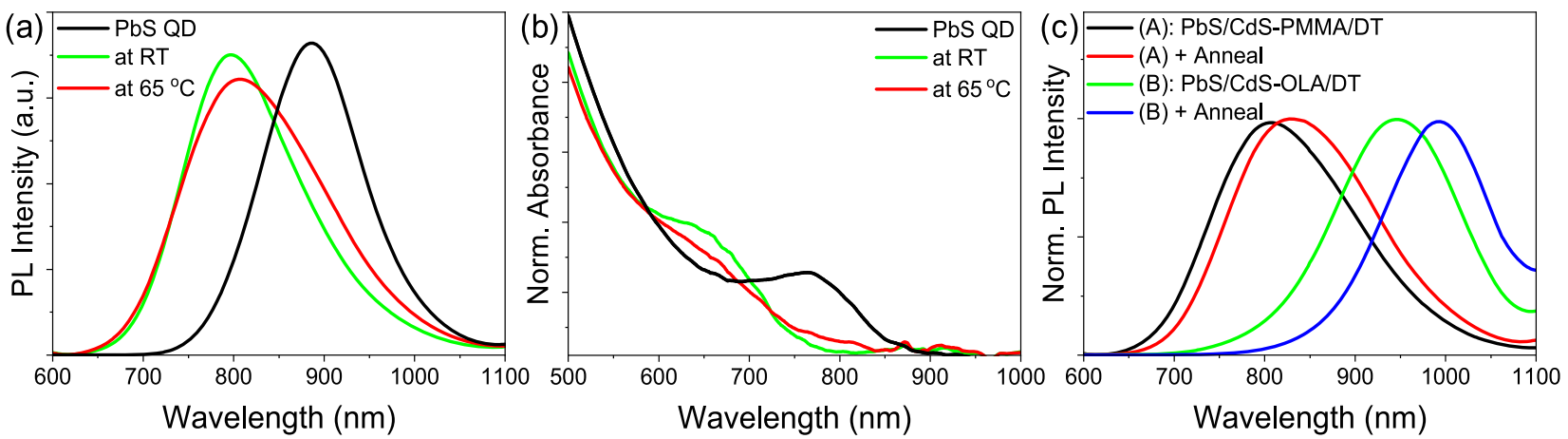

Figure 4. (a) Absorbance-calibrated PL. (b) Absorbance spectra of PbS/CdS-PMAA QDs prepared via cation exchange at room temperature and $65{ }^{\circ} \mathrm{C}$ at identical stoichiometries and reaction durations. (c) Comparison of the emission peak before and after thermal annealing of $\mathrm{PbS} / \mathrm{CdS}-$ PMAA (black and red curves) and PbS/CdS-OLA (green and blue curves) QDs.

by digestive ripening. ${ }^{27,28}$ Strong ligand-metal interaction breaking apart the particles, producing smaller ones so that more ligands can be bound to the surface, is suggested as the possible cause. ${ }^{27}$ Hence, the interaction of lead with thiols may enhance the dissolution of surface $\mathrm{Pb}$ ions and achieve a thermodynamically feasible state to enhance digestive ripening. ${ }^{28}$ Parallel to this, the removal of defects originating from adatoms during digestive ripening may be suggested as the cause of subsequent enhancement in the PL intensity. However, at lower ratios of Pb:PMAA (1:1.2), where already smaller particles were formed at the initial state, such an effect has been lost (Figure S3b). This may be due to better stability of the smaller $\mathrm{PbS}$ QDs, steric hindrance of PMAA, or a relatively higher ratio of PMAA:DT. ${ }^{29}$

Influence of the PMAA Molecular Weight on the Particle Properties. The influence of the polymer molecular weight was also investigated via reactions performed at the same mole ratio of Pb:S:OLA:PMAA but with two different PMAA samples (5 and $131 \mathrm{kDa}$ ). High-molecular-weight PMAA provided 2.5 times stronger emission intensity compared to low-molecular-weight PMAA but with no significant change in the peak positions of the QDs (Figure S3c). This observation may indicate a couple of things: (1) at this Pb:PMAA ratio, the crystal size is not affected further by the PMAA chain length because we have small OLA and DT in these reactions; $(2)$ the increased amount of carboxylic acid units per polymer chain provides better surface passivation because of freedom of mobility at distant repeat units of the polymer chain. Hence, it seems like the molecular weight of the polymer under these experimental conditions did not play a critical role in the crystal size but contributed to surface passivation significantly.

Influence of DT on the Particle Properties. PMAA and DT constitute the major components of our proposed hybrid coating. Parts a and b of Figure 3 compare the PL spectrum of $\mathrm{PbS}$ QDs prepared with $(1 \mathrm{~mL}$ of DT) and without DT in the reaction mixture, pointing out the beneficial presence of DT. All QDs produced with DT have a PL peak position between 850 and $950 \mathrm{~nm}$ throughout $30 \mathrm{~min}$ of crystal growth (Figure 3a), but those produced without DT have larger crystals with emission maxima mostly above $1100 \mathrm{~nm}$ after the first minute of the reaction (Figure $3 \mathrm{~b}$ ). DT binds to the QD surface via a $\mathrm{Pb}-\mathrm{S}$ bond much more strongly than OLA or PMAA and provides relatively stable surface passivation, which reduces the size and enhances the emission intensity. Specifically, the addition of $1 \mathrm{~mL}$ of DT caused a $152 \mathrm{~nm}$ blue shift in the emission peak maxima with ca. 3.5 times stronger intensity
(Figure 3c). The amount of DT has a moderate impact on the optical properties of QDs. Reducing the DT amount to $0.5 \mathrm{~mL}$ caused a slight red shift in the peak maxima with a $50 \%$ reduction in the intensity (Figure 3d,e). This was accompanied by a red shift in the excitonic peak, as expected (Figure $3 \mathrm{e}$ ). Increasing the DT amount above $1 \mathrm{~mL}$ did not cause any further size reduction or intensity enhancement; therefore, the addition of $1.0 \mathrm{~mL}$ of DT was determined as the best amount to be used in further reactions. Collective data indicate that the synergetic effect of hybrid DT and PMAA in the coating composition is necessary to achieve strongly luminescent and ultrasmall colloidal $\mathrm{PbS}$ QDs with emission below $1100 \mathrm{~nm}$. Because DT improves the solubility of $\mathrm{S}$ as well, $\mathrm{PbS}$ synthesis with a hybrid coating mixture was also performed at $65{ }^{\circ} \mathrm{C}$ to slow the reaction and produce smaller particles. This provided strongly luminescent QDs with emission maxima at ca. 894 $\mathrm{nm}$.

3.2. Development and Characterization of $\mathrm{PbS} / \mathrm{CdS}$ QDs. In order to improve the stability and luminescence intensity of $\mathrm{PbS} \mathrm{QDs}$, a thin $\mathrm{CdS}$ shell was grown via a room temperature cation-exchange process (Figure 4a). ${ }^{14}$ TEM micrographs show no significant difference in the crystal size (Figure $1 \mathrm{a}, \mathrm{b}$ ), which is quite expected for such a process. It is not possible to differentiate the $\mathrm{CdS}$ layer from these images because $\mathrm{Cd}$ exchanges $\mathrm{Pb}$ only from a few atomic layers from the surface at low temperatures. ${ }^{14,30-32}$ The CdS shell thickness was estimated from the shift of the absorbance of QDs after shell deposition (from 767 and $653 \mathrm{~nm}$; Figure 4b). By using the sizing curve (provided in the Supporting Information), we estimate a 0.175 -nm-thick $\mathrm{CdS}$ shell, which roughly corresponds to two layers of $\mathrm{CdS}$.

Performing cation exchange at $65{ }^{\circ} \mathrm{C}$ instead of room temperature did not cause a significant difference in the optical properties of the $\mathrm{PbS} / \mathrm{CdS}$ QDs (Figure 4a,b). This is important to note as an effect of PMAA on the cationexchange process because the same process in the absence of PMAA was shown to be temperature-dependent. ${ }^{11,14}$ A hybrid coating provided a smaller blue shift and a thinner CdS shell on $\mathrm{PbS}$ compared to just a OLA/DT coating. ${ }^{14}$ These indicate better surface quality and stability in the presence of PMAA on the core $\mathrm{QD}$. As a result, PbS/CdS-PMAA QDs emitting strongly at $790 \mathrm{~nm}(2.28 \mathrm{~nm})$ were produced. PbS/CdSPMAA QDs were subjected to mild annealing to increase the crystallinity. Annealing resulted in a $24 \mathrm{~nm}$ red shift in the emission peak of these QDs, which is about 50\% less than the shift seen in the case of $\mathrm{PbS} / \mathrm{CdS}$ without PMAA in the 

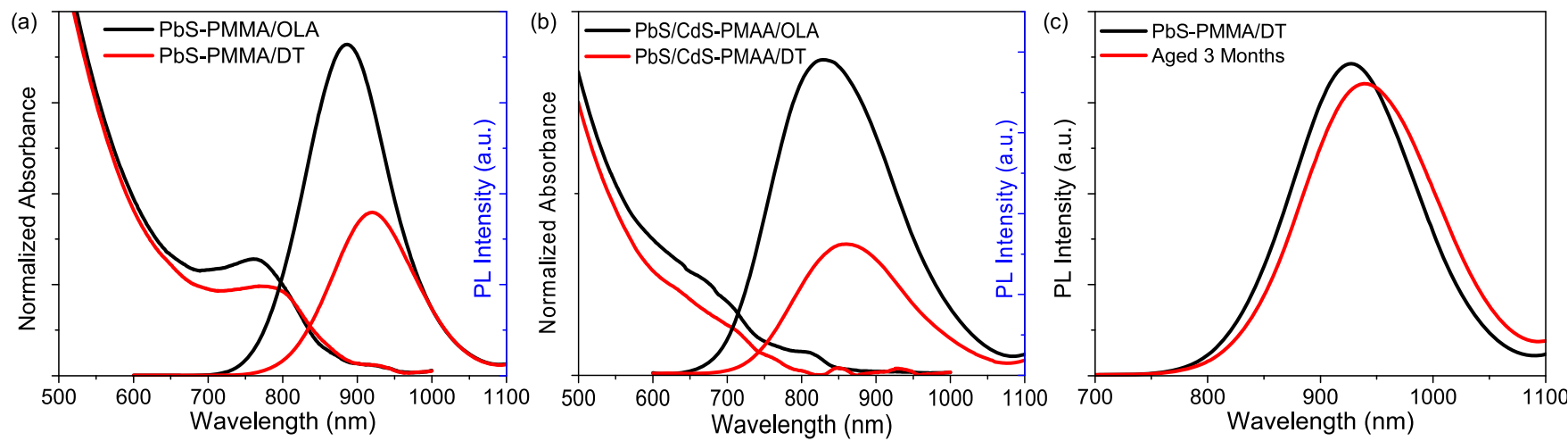

Figure 5. PL and absorbance spectra of (a) PbS-PMAA/OLA and (b) PbS/CdS-PMAA/OLA before and after DT ligand exchange. (c) Stability of $\mathrm{PbS}$-PMAA/DT produced at a Pb:PMAA ratio of 1:0.8 after 3 months in chloroform.
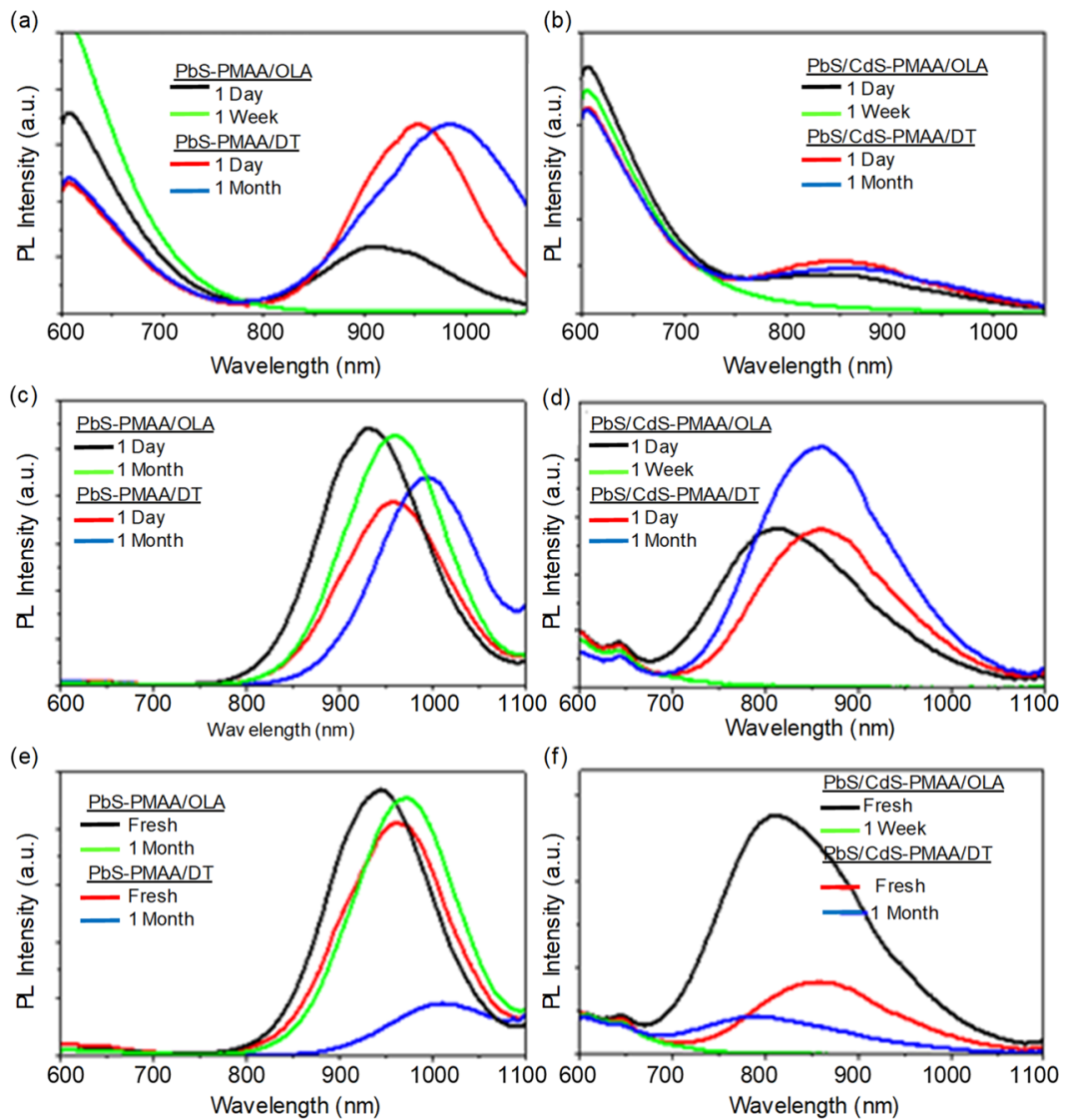

Figure 6. Time-dependent changes in the emission of PbS-PMAA/X and PbS/CdS-PMAA/X (X = OLA, DT) QDs in (a and b) oil, (c and d) gasoline, and (e and f) diesel.

coating, further suggesting higher temperature stability with a PMAA coating (Figure 4c). This was expected because of multidentate binding of the polymer. Annealed QDs show better long-term stability, which will be discussed later.

3.3. Ligand Exchange and Stability of the Particles. The exchange of relatively weakly bound OLA or OA with thiolated ligands such as DT usually enhances the stability of QDs because of stronger bonding between $\mathrm{Pb}$ and $\mathrm{S}$ atoms. ${ }^{33}$ Therefore, a postsynthetic ligand-exchange process is usually applied to improve the resistance of QDs against photodegradation/photooxidation during storage. ${ }^{14,19}$ The same strategy was evaluated for PbS-PMAA and PbS/CdS-PMAA QDs. The exchange of a heavy PMAA chain, which probably adsorbed to the crystal surface from multiple sites, or DT, which is already strongly bound to the crystal surface, is not expected, but the exchange of OLA with DT was confirmed in our previous study. ${ }^{19}$ In line with our previous observation, postsynthetic ligand exchange with DT caused 37 and $20 \mathrm{~nm}$ red shifts in the emission maxima of $\mathrm{PbS}$ and $\mathrm{PbS} / \mathrm{CdS}$ QDs, respectively (Figure 5a,b), which is less than the shifts observed for QDs without PMAA in the coating. ${ }^{14}$ This may indicate less OLA in the polymer containing a coating and/or limited diffusion of DT to the surface. Yet, DT exchange still resulted in a reduction in the emission intensity, as usual. ${ }^{34,35}$ However, the major advantage of DT ligand exchange is longterm stability. Here, the lack of a dramatic change in the original peak position and the emission intensity as well as maintenance of colloidal stability are monitored for long-term 
stability. QDs prepared with Pb:PMAA ratios of 1:0.4 and 1:0.8 showed only 38 and $11 \mathrm{~nm}$ red shifts with $11.8 \%$ and $6.4 \%$ loss in intensity after 3 months in chloroform, respectively. This proves that better particles were obtained with higher amounts of PMAA in the coating, which enhances not only the emission intensity but also the stability over time. Actually, in the absence of PMAA in the coating, the corresponding PbS-DT QDs show a larger red shift with a bigger drop in the luminescence intensity compared to those of PbS-PMAA/DT (Figure S5a,c). So, PMAA in the coating of $\mathrm{PbS}$ provided a dramatic enhancement in the stability, and after 3 months in chloroform, the luminescence of $\mathrm{PbS}$ PMAA/DT is very similar to that of the fresh sample. In the case of $\mathrm{PbS} / \mathrm{CdS}-\mathrm{DT}$, an enhancement in the luminescent intensity coupled with a significant red shift in the peak position was detected in 3 months. However, $\mathrm{PbS} / \mathrm{CdS}$ PMAA/DT prevented the peak shift while providing an even larger increase in the intensity over 3 months in chloroform (Figure S5c,d). Overall, PMAA is very effective in maintaining the original peak position of both $\mathrm{PbS}$ and $\mathrm{PbS} / \mathrm{CdS} \mathrm{QDs}$.

3.4. Stability of $\mathrm{PbS}$ and $\mathrm{PbS} / \mathrm{CdS}$ QDs in Liquid Petroleum Products. As stated earlier, in order to have practical QD-based nanotags for petroleum products, strong emission between 700 and $1000 \mathrm{~nm}$ with long-term colloidal and optical stability is necessary. ${ }^{10}$ It is also important to have a strong signal with low levels of nanotags, i.e., at the parts per million level. This requires no or a small change in the position and intensity of the emission during the lifetime of the liquid. For especially gasoline and diesel, a stability for about 1 month is sufficient, but longer is better for oil. Most petroleum products contain chemically active, classified additives to improve the combustion efficiency, which often causes fast quenching of luminescence if commercial $\mathrm{PbS}$ is used. Here, we first evaluated the optical signal generation and stability of $\mathrm{PbS}$ and $\mathrm{PbS} / \mathrm{CdS} \mathrm{QDs}$ with PMAA in the coating composition, before and after exchange of OLA with DT in oil, diesel, and gasoline (Figure 6, Tables S1-S3, and Figures S6-S8). In order to evaluate the impact of PMAA on longterm stability, the emission properties of $\mathrm{PbS}$ and $\mathrm{PbS} / \mathrm{CdS}$ after DT ligand exchange were also studied. All liquids were marked with $100 \mathrm{ppm}$ QD. This is indeed very challenging with noncustomized commercial $\mathrm{PbS}$, which undergoes fast degradation as aresult of desorption of ligands from the surface in dilute solutions.

Stability in Oil. Both PbS-PMMA and PbS/CdS-PMAA lost luminescence in oil in 1 week. In addition, the strong emission of oil at around $600 \mathrm{~nm}$ is stronger than the luminescence intensity of $100 \mathrm{ppm}$ QDs (Figure 6a,b). Active phosphine additives that are frequently used as efficiency enhancers in oils may perturb the QD surface, causing degradation or defect formation, and, hence, may cause luminescence quenching. On the other hand, DT exchange of OLA from the coating composition provided stronger emissions for both $\mathrm{PbS}$ and $\mathrm{PbS} / \mathrm{CdS}$ in oil and prevented luminescence quenching, which is a significant improvement (Figure 6a,b). In the case of PbSPMAA/DT QDs, a tolarable red shift $(31 \mathrm{~nm})$, accompanied by no change in the emission intensity over 1 month, was observed (Figure 6a). PbS/CdS-PMAA/OLA QDs maintained the peak position with only $13 \%$ loss in the emission intensity (Figure 6b). PbS-DT showed a larger drop in the emission intensity (36\%; Figure S6a), and PbS/CdS-DT showed a greater blue shift $(28 \mathrm{~nm})$ in the peak position compared to analogous structures with PMAA (Figure S6a,b and Table S1).
However, the most dramatic advantage of PMAA on the stability of QDs in oil was seen at even longer storage, which is highly desirable: Luminescence of PbS-DT quenched completely after the first month, while PbS-PMAA/DT displayed emission as strong as that of the fresh sample even after 6 months with a small red shift in the peak maxima (Figure S6ac). In the case of $\mathrm{PbS} / \mathrm{CdS}$, luminescence did not change significantly after 6 months with the polymeric coating, while a dramatic drop in the intensity was recorded in the first 4 months of PbS/CdS-DT (Figure S6b-d). Overall, PMAAcoating-coupled DT ligand exchange provides luminescence for over 6 months, for the time being, in the oil with acceptable small changes or no changes in the emission peak position and/or intensity, and a CdS shell minimizes the shift of the peak position.

Stability in Gasoline. PbS-PMAA/OLA QDs showed some spectral shift (31 nm red shift) with almost no loss in the emission intensity (3\%) after 1 month (Figure 6c), which is quite acceptable. On the other hand, PbS/CdS-PMAA/OLA QDs quenched completely in 1 week, but exchange of OLA with DT provided the expected stabilization (Figure 6d): $34 \%$ increase in the emission intensity with only a $5 \mathrm{~nm}$ red shift after 1 month of storage in gasoline. The emission at around $650 \mathrm{~nm}$ originates from the gasoline. DT ligand exchange improved the emission intensity of PbS-PMAA by $12 \%$, with a $16 \mathrm{~nm}$ red shift observed. So, DT ligand exchange reduced the spectral shift for both core and core-shell QDs with improved emission intensity and stability.

In comparison, $\mathrm{PbS}-\mathrm{DT}$ and $\mathrm{PbS} / \mathrm{CdS}-\mathrm{DT}$ exhibit losses in their emission intensities (23 and 13\%, respectively) after 1 month, while their PMAA-containing analogues (PbS-PMAA/ $\mathrm{DT}$ and $\mathrm{PbS} / \mathrm{CdS}-\mathrm{PMAA} / \mathrm{DT}$ ) demonstrate increasing emission intensity within 4 months with a small red shift in the case of $\mathrm{PbS}$ QDs, which is prevented further by the coreshell structure (Figure S7 and Table S2). So, especially PbS/ CdS-PMAA/DT is quite promising for tagging gasoline.

Stability in Diesel. The PL emission intensity of PbSPMAA/OLA QDs was reduced by $3.2 \%$, and its emission peak was shifted $29 \mathrm{~nm}$ to longer wavelengths if stored 1 month in diesel (Figure 6e and Table S3). After the first month, PbSPMAA/OLA QDs experienced a larger red shift, accompanied by a significant loss in intensity, but there is still strong emission even at the fourth month in diesel (Figure S8c). Exchange of OLA with DT (PbS-PMAA/DT QDs) resulted in a larger $(49 \mathrm{~nm})$ red shift and a dramatic drop in the emission intensity $(77.6 \%)$, yet this is slightly better than PbS-DT QDs, which almost completely quenched in 1 month with ca. 105 $\mathrm{nm}$ red shift (Figure 6e, Table S3, and Figure S8a). Hence, PMAA seems to play a major role in the stabilization of $\mathrm{PbS}$ QDs in diesel. In the absence of PMAA, the CdS shell provides some stability to $\mathrm{PbS}$ QDs: PbS/CdS-DT QDs experienced only a $25.3 \%$ loss in the emission intensity with a much smaller $(17 \mathrm{~nm})$ blue shift (Figure S8b). In the case of core-shell QDs with PMAA, the emission of PbS/CdS-PMAA/OLA diminished after 1 week, but PbS/CdS-PMAA/DT showed a large $(75 \mathrm{~nm})$ blue shift with a $48.9 \%$ drop in the emission intensity over 1 month (Figure 6f).

Overall, diesel stands out as an exceptional medium, which is very harsh on the particles. However, PbS-PMAA/OLA provided an acceptable performance.

3.5. Development of Binary Nanotags. Previously, we have developed a method and device to detect such optical barcodes in liquids during flow. ${ }^{10}$ Although a single QD can be 

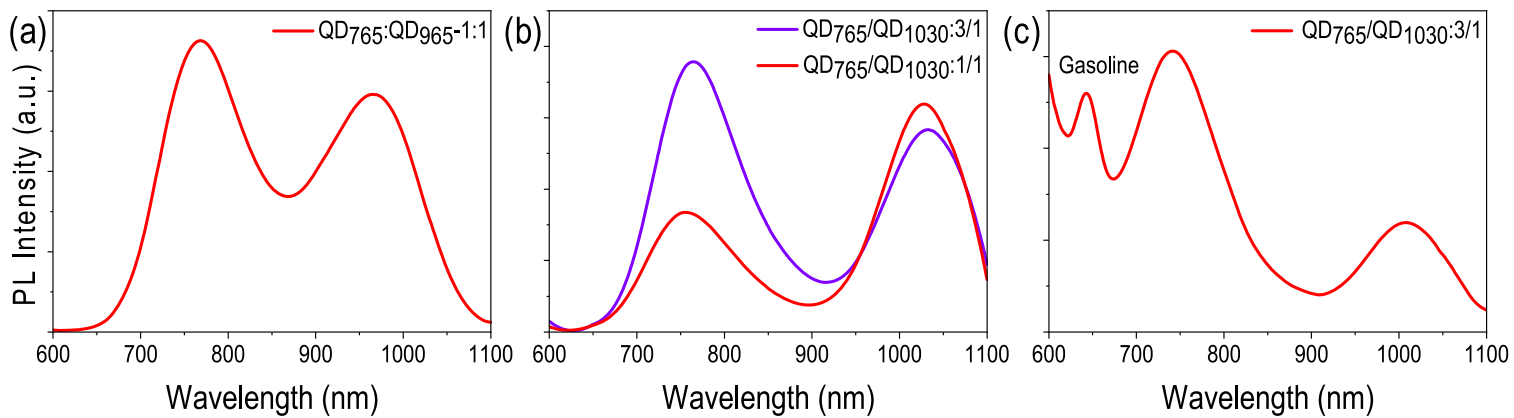

Figure 7. PL spectra of barcodes created with (a) $\mathrm{QD}_{765} / \mathrm{QD}_{965}$ and (b) $\mathrm{QD}_{765} / \mathrm{QD}_{1030}$ in chloroform and (c) $\mathrm{QD}_{765} / \mathrm{QD}_{1030}$ in gasoline.

enough to tag a product, a barcode would be more advanced and difficult to imitate. In order to create an optical nanotag in the form of a barcode, several QDs with different emission wavelengths would be necessary. In theory, the possible number of barcodes could be $x y-1$, where $x$ is the number of different emission intensities and $y$ is the number of QDs with significantly different emission wavelengths. ${ }^{7}$ Hence, changes in the peak positions and intensities of the QDs used as nanotags may not be very important if a single QD is used but would be an important limitation if a barcode is prepared.

In Figure 7a, we used two different PbS-PMAA/DT QDs with emission maxima at $765 \mathrm{~nm}\left(\mathrm{QD}_{765}\right)$ and $965 \mathrm{~nm}$ $\left(\mathrm{QD}_{965}\right)$ in chloroform to create a barcode. Figure $7 \mathrm{~b}$ shows the emission of two different barcodes created with $\mathrm{QD}_{765}$ and $\mathrm{QD}_{1030}$ at two different ratios, keeping the concentration of $\mathrm{QD}_{1030}$ constant, in chloroform. The emission intensity of $\mathrm{QD}_{1030}$ was reduced by ca. $11 \%$ when the ratio of $\mathrm{QD}_{765} /$ $\mathrm{QD}_{1030}$ changed from 1 to 3 . Parallel to this, the increase in the emission intensity of $\mathrm{QD}_{765}$ seemed to be about $24.5 \%$, which is less than expected. Here, the same numbers of photons were absorbed by twice as many QDs in the later case, reducing the number of photons per particle. It may also be suggested that Förster resonance energy transfer (FRET) could contribute to the emission intensity of $\mathrm{QD}_{1030}$ as well. ${ }^{36}$ When the ratio of small to larger QDs was increased to 5, we saw the same trend (Figure S9). Therefore, adjusting the QD concentrations for about $25 \%$ difference in the emission intensity seems safe in creating optical barcodes with these QDs, at least for the binary ones.

A successful optical barcode was also demonstrated in gasoline with $\mathrm{QD}_{765}$ and $\mathrm{QD}_{1030}$ (Figure $7 \mathrm{c}$ ). The use of nanotags in liquid petroleum products has its own specific limitations because of the strong autofluorescence of these materials. Therefore, all QDs produced here were designed to provide a strong emission above $700 \mathrm{~nm}$. In addition, absorbance and emission of the petroleum products would affect the real emission intensity of each QD for a specific concentration in the barcode, as discussed above. Basically, petroleum products would be acting as another fluorophore. It is also known that the medium influences the luminescence due to solvent-QD interaction, which is important here as well. For example, changing the medium from chloroform to gasoline shifted the emission maxima of both $\mathrm{QD}_{765}$ and $\mathrm{QD}_{1030}$ by ca. $25-30 \mathrm{~nm}$ to shorter wavelengths. Thus, all of these features need to be considered in deciding the difference in the peak maxima of different QDs and the number of different concentrations that can be used in tagging a specific liquid with a QD-based optical barcode.

\section{CONCLUSIONS}

Ultrasmall $\mathrm{PbS}$ and $\mathrm{PbS} / \mathrm{CdS}$ QDs with strong emission between 700 and $1100 \mathrm{~nm}$ were prepared using a PMAA/DT hybrid coating system at low reaction temperatures (below 100 ${ }^{\circ} \mathrm{C}$ ) using safe precursors in a relatively greener synthetic route. PMAA provided some size tuning but, more importantly, smaller $\mathrm{PbS}$, which got even smaller with much stronger luminescence intensity when DT was added in situ. The use of PMAA in the hybrid coating provided the desired long-term stability to these QDs, which was not achieved otherwise. The presence of PMAA in the coating had an important impact on the changes observed after $\mathrm{CdS}$ deposition and DT ligand exchange, indicating significant stabilization of $\mathrm{PbS}$ with PMAA.

The influences of the PMAA core-shell structure and postsynthetic ligand exchange on the QD stability were tested in chloroform and petroleum products, namely, gasoline, diesel, and oil. In chloroform, all particles showed great stability for the 3 months that were monitored, with mostly acceptable spectral changes. In petroleum products, (i) PMAA is quite effective in maintaining the position of the emission peak because it provides better resistance against oxidation and Ostwald ripening, (ii) DT ligand exchange in a postsynthesis process is essential for most $\mathrm{PbS}$ and $\mathrm{PbS} / \mathrm{CdS} \mathrm{QDs}$ for stability, (iii) a CdS shell provides better resistance to photodegradation almost in all petroleum products in the absence of PMAA in the coating, (iv) PMAA coating with DT ligand exchange provides better stability in oil and gasoline (up to 6 months), and (v) although diesel is quite harsh on these QDs, PMAA provides sufficient stability. Hence, appropriate QD compositions for each of these petroleum products with detectable, strong luminescence were produced as optical tags.

Successful binary optical barcodes in the NIR region were also demonstrated at the parts per million levels using a lowcost silicon detector and the stable QDs produced here. This is quite a unique feature of NIR QDs as optical barcodes in luminescent solvents and is the first example of such an application in the literature, which can probably guide future studies in the field.

\section{ASSOCIATED CONTENT}

\section{Supporting Information}

The Supporting Information is available free of charge at https://pubs.acs.org/doi/10.1021/acsanm.9b01780.

Further details of the description of QDs, size calculation, stability data, barcode generation, and TEM images (PDF) 


\section{AUTHOR INFORMATION}

Corresponding Author

*Email: fyagci@ku.edu.tr.

ORCID $\odot$

Emek G. Durmusoglu: 0000-0001-6840-8342

Yurdanur Turker: 0000-0003-3611-6243

Havva Yagci Acar: 0000-0001-5601-8814

\section{Present Address}

${ }^{\dagger}$ E.G.D.: LUMINOUS! Centre of Excellence for Semiconductor Lighting and Displays, School of Electrical and Electronic Engineering, Nanyang Technological University, Singapore 639798, Singapore.

\section{Funding}

This project was funded by OPET Inc. (Project OPET 201301) and KUANTAG Inc. (Project KUANTAG 2015-01).

\section{Notes}

The authors declare no competing financial interest.

\section{ACKNOWLEDGMENTS}

The authors thank Dr. Gulcan Corapcioglu and Prof. Mehmet A. Gulgun (Sabanci University, Istanbul, Turkey) for the TEM images.

\section{REFERENCES}

(1) Kim, J. Y.; Voznyy, O.; Zhitomirsky, D.; Sargent, E. H. 25th Anniversary Article: Colloidal Quantum Dot Materials and Devices: A Quarter-Century of Advances. Adv. Mater. 2013, 25, 4986-5010.

(2) Reiss, P.; Protiere, M.; Li, L. Core/Shell Semiconductor Nanocrystals. Small 2009, 5, 154-168.

(3) Van Veggel, F. C. J. M. Near-Infrared Quantum Dots and Their Delicate Synthesis, Challenging Characterization, and Exciting Potential Applications. Chem. Mater. 2014, 26, 111-122.

(4) Zhao, H.; Rosei, F. Colloidal Quantum Dots for Solar Technologies. Chem. 2017, 3, 229-258.

(5) Li, Y.; Li, Z.; Wang, X.; Liu, F.; Cheng, Y.; Zhang, B.; Shi, D. In Vivo Cancer Targeting and Imaging-Guided Surgery with Near Infrared-Emitting Quantum Dot Bioconjugates. Theranostics 2012, 2, 769-776.

(6) Duman, F. D.; Khodadust, R.; Durmusoglu, E. G.; Yagci, M. B.; Acar, H. Y. Impact of Reaction Variables and Pei/L-Cysteine Ratio on the Optical Properties and Cytocompatibility of Cationic $\mathrm{Ag}_{2} \mathrm{~S}$ Quantum Dots as NIR Bio-Imaging Probes. RSC Adv. 2016, 6, 77644-77654.

(7) Han, M.; Gao, X.; Su, J. Z.; Nie, S. Quantum-Dot-Tagged Microbeads for Multiplexed Optical Coding of Biomolecules. Nat. Biotechnol. 2001, 19, 631-635.

(8) Luther, R. Identification of Lubricants - A New Approach. MTZ. worldwide 2008, 69, 18-23.

(9) Siegel, J. A.; Fisher, J.; Gilna, C.; Spadafora, A.; Krupp, D. Fluorescence of Petroleum Products I. Three-Dimensional Fluorescence Plots of Motor Oils and Lubricants. J. Forensic Sci. 1985, 30, 741-759.

(10) Urey, H.; Yagci Acar, H.; Elbuken, C.; Can, B.; Akgun, O. V.; Uygurmen, F. K. A Method and an Apparatus for the Detection of a Tagging Material in Fluids. U.S. Patent 20160025667A1, 2016.

(11) Zhao, X.; Gorelikov, I.; Musikhin, S.; Cauchi, S.; Sukhovatkin, V.; Sargent, E. H.; Kumacheva, E. Synthesis and Optical Properties of Thiol-Stabilized PbS Nanocrystals. Langmuir 2005, 21, 1086-1090.

(12) Justo, Y.; Geiregat, P.; Van Hoecke, K.; Vanhaecke, F.; De Mello Donega, C.; Hens, Z. Optical Properties of PbS/CdS Core/ Shell Quantum Dots. J. Phys. Chem. C 2013, 117, 20171-20177.

(13) Clark, P. C. J.; Radtke, H.; Pengpad, A.; Williamson, A. I.; Spencer, B. F.; Hardman, S. J. O.; Leontiadou, M. A.; Neo, D. C. J.; Fairclough, S. M.; Watt, A. A. R.; Pis, I.; Nappini, S.; Bondino, F.; Magnano, E.; Handrup, K.; Schulte, K.; Silly, M. G.; Sirotti, F.; Flavell,
W. R. The Passivating Effect of Cadmium in PbS/CdS Colloidal Quantum Dots Probed by nm-scale Depth Profiling. Nanoscale 2017, 9, 6056-6067.

(14) Durmusoglu, E. G.; Yildizhan, M. M.; Gulgun, M. A.; Yagci Acar, H. Production of Small, Stable PbS/CdS Quantum Dots Via Room Temperature Cation Exchange Followed by a Low Temperature Annealing Processes. J. Phys. Chem. C 2017, 121, 25520-25530.

(15) Zhou, N.; Chen, G. P.; Zhang, X. L.; Cheng, L. Y.; Luo, Y. H.; Li, D. M.; Meng, Q. B. Highly Efficient PbS/CdS Co-Sensitized Solar Cells Based on Photoanodes with Hierarchical Pore Distribution. Electrochem. Commun. 2012, 20, 97-100.

(16) Gonfa, B. A.; Kim, M. R.; Delegan, N.; Tavares, A. C.; Izquierdo, R.; Wu, N.; El Khakani, M. A.; Ma, D. Towards High Efficiency Air-Processed Near-Infrared Responsive Photovoltaics: Bulk Heterojunction Solar Cells Based on PbS/CdS Core-Shell Quantum Dots and $\mathrm{TiO}_{2}$ Nanorod Arrays. Nanoscale 2015, 7, 10039-10049.

(17) Sanchez, R. S.; Binetti, E.; Torre, J. A.; Garcia-Belmonte, G.; Striccoli, M.; Mora-Sero, I. All Solution Processed Low Turn-on Voltage Near Infrared Leds Based on Core-Shell PbS-CdS Quantum Dots with Inverted Device Structure. Nanoscale 2014, 6, 8551-8555.

(18) Saran, R.; Curry, R. J. Lead Sulphide Nanocrystal Photodetector Technologies. Nat. Photonics 2016, 10, 81-92.

(19) Durmusoglu, E. G.; Turker, Y.; Acar, H. Y. Green Synthesis of Strongly Luminescent, Ultrasmall $\mathrm{PbS}$ and PbSe Quantum Dots. J. Phys. Chem. C 2017, 121, 12407-12415.

(20) Kas, R.; Sevinc, E.; Topal, U.; Acar, H. Y. A Universal Method for the Preparation of Magnetic and Luminescent Hybrid Nanoparticles. J. Phys. Chem. C 2010, 114, 7758-7766.

(21) Huang, Z.; Zhai, G. M.; Zhang, Z. M.; Zhang, C. W.; Xia, Y.; Lian, L. Y.; Fu, X. M.; Zhang, D. L.; Zhang, J. B. Low Cost and Large Scale Synthesis of PbS Quantum Dots with Hybrid Surface Passivation. CrystEngComm 2017, 19, 946-951.

(22) Cademartiri, L.; Bertolotti, J.; Sapienza, R.; Wiersma, D. S.; von Freymann, G.; Ozin, G. A. Multigram Scale, Solventless, and Diffusion-Controlled Route to Highly Monodisperse PbS Nanocrystals. J. Phys. Chem. B 2006, 110, 671-673.

(23) Zhang, J.; Gao, J.; Miller, E. M.; Luther, J. M.; Beard, M. C. Diffusion-Controlled Synthesis of $\mathrm{PbS}$ and $\mathrm{PbSe}$ Quantum Dots with In Situ Halide Passivation for Quantum Dot Solar Cells. ACS Nano 2014, 8, 614-622.

(24) Xu, Y.; Lin, Y.; Zhuang, L.; Lin, J.; Lv, J.; Huang, Q.; Sun, J. Bleomycin Loaded Magnetite Nanoparticles Functionalized by Polyacrylic Acid as a New Antitumoral Drug Delivery System. BioMed Res. Int. 2013, 2013, 1-5.

(25) Zhao, H. G.; Chaker, M.; Wu, N. Q.; Ma, D. L. Towards Controlled Synthesis and Better Understanding of Highly Luminescent $\mathrm{PbS} / \mathrm{CdS}$ Core/Shell Quantum Dots. J. Mater. Chem. 2011, 21, 8898-8904.

(26) Moreels, I.; Justo, Y.; De Geyter, B.; Haustraete, K.; Martins, J. C.; Hens, Z. Size-Tunable, Bright, and Stable PbS Quantum Dots: A Surface Chemistry Study. ACS Nano 2011, 5, 2004-2012.

(27) Zhao, H.; Chaker, M.; Ma, D. Bimodal Photoluminescence During the Growth of PbS Quantum Dots. J. Phys. Chem. C 2009, 113, 6497-6504.

(28) Prasad, B. L. V.; Stoeva, S. I.; Sorensen, C. M.; Klabunde, K. J. Digestive Ripening of Thiolated Gold Nanoparticles: The Effect of Alkyl Chain Length. Langmuir 2002, 18, 7515-7520.

(29) Choi, H.; Ko, J. H.; Kim, Y. H.; Jeong, S. Steric-HindranceDriven Shape Transition in PbS Quantum Dots: Understanding SizeDependent Stability. J. Am. Chem. Soc. 2013, 135, 5278-81.

(30) Beberwyck, B. J.; Surendranath, Y.; Alivisatos, A. P. Cation Exchange: A Versatile Tool for Nanomaterials Synthesis. J. Phys. Chem. C 2013, 117, 19759-19770.

(31) Justo, Y.; Sagar, L. K.; Flamee, S.; Zhao, Q.; Vantomme, A.; Hens, Z. Less Is More. Cation Exchange and the Chemistry of the Nanocrystal Surface. ACS Nano 2014, 8, 7948-7957.

(32) Zhang, H.; Selopal, G. S.; Zhou, Y.; Tong, X.; Benetti, D.; Jin, L.; Navarro-Pardo, F.; Wang, Z.; Sun, S.; Zhao, H.; Rosei, F. 
Controlled Synthesis of Near-Infrared Quantum Dots for Optoelectronic Devices. Nanoscale 2017, 9, 16843-16851.

(33) Zhang, J.; Wang, Y.; Zheng, J.; Huang, F.; Chen, D.; Lan, Y.; Ren, G.; Lin, Z.; Wang, C. Oriented Attachment Kinetics for Ligand Capped Nanocrystals: Coarsening of Thiol-PbS Nanoparticles. J. Phys. Chem. B 2007, 111, 1449-1454.

(34) Zhao, H.; Liang, H.; Gonfa, B. A.; Chaker, M.; Ozaki, T.; Tijssen, P.; Vidal, F.; Ma, D. Investigating Photoinduced Charge Transfer in Double- and Single-Emission PbS@CdS Core@Shell Quantum Dots. Nanoscale 2014, 6, 215-225.

(35) Jeong, S.; Achermann, M.; Nanda, J.; Ivanov, S.; Klimov, V. I.; Hollingsworth, J. A. Effect of the Thiol-Thiolate Equilibrium on the Photophysical Properties of Aqueous CdSe/ZnS Nanocrystal Quantum Dots. J. Am. Chem. Soc. 2005, 127, 10126-10127.

(36) Chou, K. F.; Dennis, A. M. Forster Resonance Energy Transfer between Quantum Dot Donors and Quantum Dot Acceptors. Sensors 2015, 15, 13288-13325. 